\title{
Joint Trajectory and Beamforming Optimization for Secure UAV Transmission Aided by IRS
}

\author{
Xiaowei Pang ${ }^{\dagger}$, Nan Zhao ${ }^{\dagger}$, Jie Tang ${ }^{\amalg}$, Dusit Niyato ${ }^{\ddagger}$ and Kai-Kit Wong* \\ ${ }^{\dagger}$ School of Information and Communication Engineering, Dalian University of Technology, Dalian, P. R. China \\ ${ }^{\amalg}$ School of Electronic and Information Engineering, South China University of Technology, Guangzhou, China \\ ${ }^{\ddagger}$ School of Computer Science and Engineering, Nanyang Technological University, Singapore \\ ${ }^{\star}$ Department of Electronic and Electrical Engineering, University College London, London WC1E 6BT, U.K.
}

\begin{abstract}
Unmanned aerial vehicle (UAV) communications are susceptible to eavesdropping, and intelligent reflecting surface (IRS) is capable of reconfiguring the propagation environment, thereby facilitating the security for UAV networks. In this paper, we aim to maximize the average secrecy rate for an IRSassisted UAV network by jointly optimizing the UAV trajectory, the transmit beamforming, and the phase shift of IRS. The complicated problem is decomposed into three sub-problems which are solved alternatively via an iterative algorithm. First, the closed-form solution to the active beamforming is derived. Then, the passive beamforming problem of fractional programming is converted into corresponding parametric sub-problems. Furthermore, the non-convex trajectory optimization problem is reformulated as a convex one by utilizing the successive convex approximation. Simulation results are provided to validate the effectiveness of the proposed scheme.

Index Terms-Beamforming optimization, intelligent reflecting surface, trajectory design, unmanned aerial vehicle.
\end{abstract}

\section{INTRODUCTION}

The advancement of unmanned aerial vehicles (UAVs) has motivated a plethora of applications of UAVs in military, civilian and commercial domains [1], including aerial inspection, cargo transport, search and rescue, video steaming, etc. Specifically, thanks to high maneuverability and cost-effective deployment, UAVs are gaining popularity in serving as aerial communication platforms to enhance the coverage, capacity and energy efficiency of existing wireless networks [2]-[4]. On the other hand, integrating UAVs into cellular networks as aerial users is envisioned to yield significant performance enhancement in network reliability and throughput [5]. In particular, UAVs are more likely to establish line-of-sight (LoS) wireless links [6], resulting in better air-ground communication quality.

Despite the appealing advantages, UAV communication networks still face many new challenges. Compared to airair wireless links, air-ground channels are more prone to blockages caused by obstacles and mountainous terrain, which restrains the performance of UAV systems [7]. Another crucial issue is that the UAV broadcasting channels are more easily exposed to eavesdropping, which thus has attracted extensive concern on the secure UAV transmission.

Nan Zhao is the corresponding author (zhaonan@dlut.edu.cn).
It is worth noting that intelligent reflecting surface (IRS) is a promising solution to address these issues, due to its capability of restructuring wireless propagation environment [8]. Composed of a large array of reflecting elements, IRS can reflect the incident signal passively to either enhance the desired signal or suppress the interference with no need of any radio-frequency (RF) chains [9].

Motivated by the appealing advantages of IRS, it has attracted significant attention to be integrated into existing UAV networks to further unleash the air-ground network potential [10]. There are extensive research on joint designs for IRS-assisted UAV communication networks. For example, in [11], Li et al. jointly optimized the UAV trajectory and the IRS passive beamforming, intending to maximize the average achievable rate. In addition, Ge et al. employed multiple IRSs to assist UAV communications in [12], where the UAV trajectory, active beamforming and passive beamforming were jointly optimized to maximize the received power at the user. The joint design of UAV trajectory, IRS scheduling, and resource allocation was investigated in [13] by Wei et al. to maximize the system sum rate. Furthermore, a novel secure IRS-assisted UAV system was presented in [14], and the secrecy rate was maximized via the joint design of trajectory, transmit power and phase shifters.

Different from the existing studies that consider either single-antenna UAVs or static UAVs, we aim at designing a secure transmission scheme for the IRS-assisted UAV network, with the mobility of UAV and multi-antenna beamforming gain both exploited. Specifically, we aim to maximize the average achievable secrecy rate by jointly optimizing the transmit beamforming and the trajectory for the UAV and the passive beamforming for the IRS. The original problem is intractable and hence is decomposed into three sub-problems. An iterative algorithm is developed to solve the three subproblems in an alternating manner.

\section{System Model and Problem Formulation}

\section{A. System Model}

We consider an IRS-assisted UAV wireless network consisting of a UAV, an IRS, a legitimate user and an eavesdropper, as illustrated in Fig. 1. In particular, the direct link 


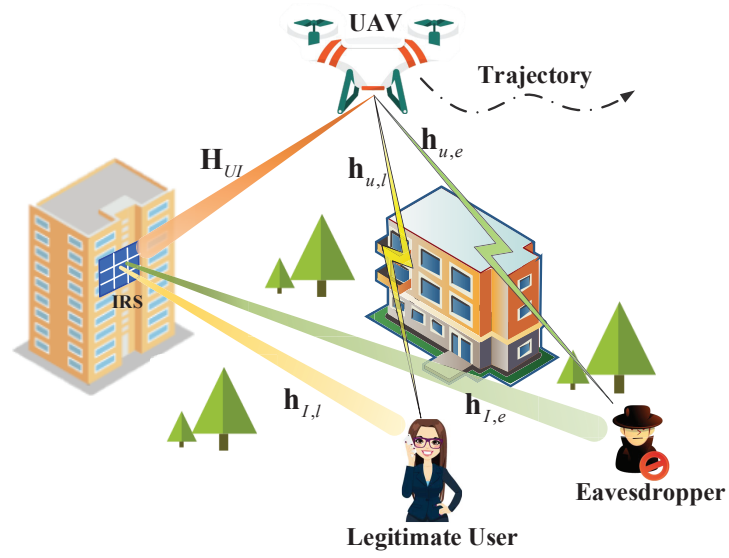

Fig. 1. Secure transmission in an IRS-assisted UAV wireless network.

between the UAV and the ground user is severely blocked by ground obstacles due to the complicated urban environment. Assume that the UAV is equipped with $N_{u}$ antennas, while both the user and the eavesdropper have a single antenna. The UAV is assumed to fly at a fixed altitude $H_{u}$ above the ground within a time period $T$. For ease of tractability, we divide $T$ into $N$ small time slots so that the location of UAV can be approximately considered unchanged in each time slot.

Without loss of generality, a 3D Cartesian coordinate system is considered, where the horizontal coordinates of the legitimate user and the eavesdropper are denoted by $\mathbf{C}_{l}=\left[x_{l}, y_{l}\right]^{T}$ and $\mathbf{C}_{e}=\left[x_{e}, y_{e}\right]^{T}$, respectively. The first element of IRS is regarded as the reference point which is assumed to be located at the horizontal location $\mathbf{C}_{I}=\left[x_{I}, y_{I}\right]^{T}$ with the height $H_{I}$. The location of UAV projected onto the ground in the $n$th time slot is denoted as $\mathbf{q}[n]=[x[n], y[n]]^{T}$, $n=1,2, \ldots, N$. The initial and final locations of UAV during the flight are given as $\mathbf{q}_{I}$ and $\mathbf{q}_{F}$, respectively. Thus, the following constraints of UAV should be satisfied.

$$
\begin{aligned}
& \mathbf{q}[1]=\mathbf{q}_{I}, \quad\left\|\mathbf{q}[N]-\mathbf{q}_{F}\right\|^{2} \leq\left(\frac{V_{m} T}{N}\right)^{2}, \\
& \|\mathbf{q}[n+1]-\mathbf{q}[n]\|^{2} \leq\left(\frac{V_{m} T}{N}\right)^{2}, n=1, \ldots, \mathcal{N}-1,
\end{aligned}
$$

where $V_{m}$ is the maximum speed of UAV.

Assume that the IRS consists of a uniform planar array (UPA) with $M=M_{x} M_{z}$ reflecting elements, where $M_{x}$ and $M_{z}$ denote the number of elements along the $x$-axis and $z$-axis, respectively. The phase shift matrix is denoted as $\boldsymbol{\Phi}[n]=\operatorname{diag}\left(e^{j \varphi_{1}[n]}, \ldots, e^{j \varphi_{m}[n]}, \ldots, e^{j \varphi_{M}[n]}\right)$, where $\varphi_{m}[n]$ represents the phase shift incurred by the $m$ th reflecting element in the $n$th time slot.

\section{B. Channel Model}

Due to the obstacles, the direct links from the UAV to ground nodes are blocked and there are lots of scattering components. The UAV-ground channels follow Rayleigh fading, with the channel model from the UAV to the legitimate user and that to the eavesdropper given respectively as

$$
\begin{gathered}
\mathbf{h}_{u, l}^{H}[n]=\sqrt{\rho d_{u, l}^{-\alpha}[n]} \widetilde{\mathbf{h}}_{u, l}[n] \in \mathbb{C}^{1 \times N_{u},} \\
\mathbf{h}_{u, e}^{H}[n]=\sqrt{\rho d_{u, e}^{-\alpha}[n]} \widetilde{\mathbf{h}}_{u, e}[n] \in \mathbb{C}^{1 \times N_{u},}
\end{gathered}
$$

where $\rho$ represents the channel power gain at the reference distance $d_{0}=1 \mathrm{~m}, \alpha$ is the path-loss exponent, $d_{u, i}[n]=$ $\sqrt{\left\|\mathbf{q}[n]-\mathbf{C}_{i}\right\|^{2}+H_{u}^{2}}$ denotes the distance from the UAV to the secure user or the eavesdropper in the $n$th time slot, $i \in$ $\{l, e\}$. The elements of $\widetilde{\mathbf{h}}_{u, i}[n](i \in\{l, e\})$ are independent and identically distributed complex Gaussian variable with zero mean and unit variance.

The channel between the UAV and IRS is considered to be dominated by LoS links thanks to the vertical heights of UAV and IRS, which is given as

$$
\mathbf{H}_{U I}[n]=\sqrt{\rho d_{U I}^{-2}[n]} \mathbf{a}_{M}^{T}[n] \mathbf{a}_{N_{U}}[n] \in \mathbb{C}^{M \times N_{u}},
$$

where $d_{U I}[n]=\sqrt{\left\|\mathbf{q}[n]-\mathbf{C}_{I}\right\|^{2}+\left(H_{u}-H_{I}\right)^{2}}$ denotes the 3D distance from the UAV to the IRS in the $n$th time slot. Particularly, $\mathbf{a}_{M}^{T}[n]$ and $\mathbf{a}_{N_{u}}[n]$ are array responses in the $n$th time slot, which are obtained in the following.

With the element spacing of IRS denoted by $\widetilde{d}_{x}$ and $\widetilde{d}_{z}$ in Axis $x$ and Axis $z$, respectively, the receive array response of IRS can be expressed as

$$
\mathbf{a}_{M}[n]=\mathbf{a}_{x}(\phi[n], \theta[n]) \otimes \mathbf{a}_{z}(\phi[n], \theta[n]),
$$

where

$$
\begin{aligned}
& \mathbf{a}_{x}(\phi[n], \theta[n])=\left[1, \ldots, e^{\left.-j \frac{2 \pi}{\lambda} \widetilde{d}_{x}\left(M_{x}-1\right) \sin \theta[n] \cos \phi[n]\right],}\right. \\
& \mathbf{a}_{z}(\phi[n], \theta[n])=\left[1, \ldots, e^{\left.-j \frac{2 \pi}{\lambda} \widetilde{d}_{z}\left(M_{z}-1\right) \cos \theta[n]\right],}\right. \\
& \sin \theta[n] \cos \phi[n]=\frac{x_{I}-x[n]}{d_{U I}[n]}, \cos \theta[n]=\frac{H_{u}-H_{I}}{d_{U I}[n]} .
\end{aligned}
$$

$\phi[n]$ denotes the azimuth angle of arrival (AoA) and $\theta[n]$ represents the elevation AoA in the $n$th time slot. $\lambda$ is the carrier wavelength.

The antennas equipped at the UAV is assumed to be a uniform linear array (ULA). With the known angle-ofdeparture $(\mathrm{AoD}) \phi_{D}[n]$ and the antenna separation $\widetilde{d}$, the transmit array response can be expressed as

$$
\mathbf{a}_{N_{U}}[n]=\left[1, e^{-j \frac{2 \pi}{\lambda} \widetilde{d} \cos \phi_{D}[n]}, \ldots, e^{-j \frac{2 \pi}{\lambda} \widetilde{d}\left(N_{u}-1\right) \cos \phi_{D}[n]}\right] .
$$

The IRS-ground channels are much more complicated and include both LoS and NLoS components. We model the channel from the IRS to a ground node $i$ as a Rician fading model, $i \in\{l, e\}$, which can be given as

$$
\mathbf{h}_{I, i}[n]=\sqrt{\rho d_{I, i}^{-\beta}}\left(\sqrt{\frac{\mathcal{K}}{\mathcal{K}+1}} \overline{\mathbf{h}}_{I, i}^{L}[n]+\sqrt{\frac{1}{\mathcal{K}+1}} \widetilde{\mathbf{h}}_{I, i}^{N}[n]\right),
$$

where $d_{I, i}$ represents the distance between the IRS and the ground node, $\beta$ is the path-loss exponent, and $\mathcal{K}$ denotes the Rician factor. With the azimuth and elevation AoDs for the LoS link from the IRS to node $i$ denoted by $\phi_{i}$ and $\theta_{i}$, the 
LoS component can be obtained as

$$
\begin{aligned}
& \overline{\mathbf{h}}_{I, i}^{L}[n]= {\left[1, \ldots, e^{-j \frac{2 \pi}{\lambda} \widetilde{d}_{x}\left(M_{x}-1\right) \sin \theta_{i} \cos \phi_{i}}\right] \otimes } \\
& {\left[1, \ldots, e^{-j \frac{2 \pi}{\lambda} \widetilde{d}_{z}\left(M_{z}-1\right) \cos \theta_{i}}\right], } \\
& \sin \theta_{i} \cos \phi_{i}=\left(x_{i}-x_{I}\right) / d_{I, i}, \quad \cos \theta_{i}=H_{I} / d_{I, i} .
\end{aligned}
$$

In addition, the NLoS component $\widetilde{\mathbf{h}}_{I, i}^{N}[n] \in \mathbb{C}^{M \times 1}$ and $\widetilde{\mathbf{h}}_{I, i}^{N}[n] \sim \mathcal{C N}(\mathbf{0}, \mathbf{I})$.

The received signal at the legitimate user and at the eavesdropper in the $n$th time slot can be modeled as

$$
\begin{aligned}
& y_{l}[n]=\left(\mathbf{h}_{u, l}^{H}[n]+\mathbf{h}_{I, l}[n] \boldsymbol{\Phi}[n] \mathbf{H}_{U I}[n]\right) \mathbf{w}[n] x[n]+z_{l}[n], \\
& y_{e}[n]=\left(\mathbf{h}_{u, e}^{H}[n]+\mathbf{h}_{I, e}[n] \boldsymbol{\Phi}[n] \mathbf{H}_{U I}[n]\right) \mathbf{w}[n] x[n]+z_{e}[n],
\end{aligned}
$$

where $x[n]$ is the transmitted signal, w $[n] \in \mathbb{C}^{N_{u} \times 1}$ denotes the beamforming vector at the UAV, and $z_{l}[n] \sim \mathcal{C N}\left(0, \sigma_{l}^{2}\right)$ and $z_{e}[n] \sim \mathcal{C N}\left(0, \sigma_{e}^{2}\right)$ denote the additive white Gaussian noise (AWGN) at the secure user and the eavesdropper.

\section{Problem Formulation}

Based on the system and channel models, the achievable rate of the legitimate user and that of the eavesdropper in the $n$th time slot can be respectively given as

$$
\begin{aligned}
& R_{L}[n]=\log _{2}\left(1+\frac{\left|\left(\mathbf{h}_{u, l}^{H}[n]+\mathbf{h}_{I, l}^{H}[n] \mathbf{\Phi}[n] \mathbf{H}_{U I}[n]\right) \mathbf{w}[n]\right|^{2}}{\sigma_{l}^{2}}\right), \\
& R_{E}[n]=\log _{2}\left(1+\frac{\left|\left(\mathbf{h}_{u, e}^{H}[n]+\mathbf{h}_{I, e}^{H}[n] \mathbf{\Phi}[n] \mathbf{H}_{U I}[n]\right) \mathbf{w}[n]\right|^{2}}{\sigma_{e}^{2}}\right) .
\end{aligned}
$$

Furthermore, the average achievable secrecy rate between the UAV and the legitimate user can be calculated as

$$
\bar{R}_{s}=\frac{1}{N} \sum_{n=1}^{N}\left(R_{L}[n]-R_{E}[n]\right) .
$$

To ensure the secure transmission, our goal is to maximize the average achievable secrecy rate by jointly optimizing the UAV trajectory $\mathbf{Q} \triangleq\{\mathbf{q}[n], \forall n\}$, the transmit beamforming $\mathbf{W} \triangleq\{\mathbf{w}[n], \forall n\}$, and the phase shift matrix $\boldsymbol{\Psi} \triangleq\{\boldsymbol{\Phi}[n], \forall n\}$. The joint optimization problem can be formulated as

$$
\begin{aligned}
\max _{\mathbf{Q}, \mathbf{W}, \mathbf{\Psi}} & \bar{R}_{s} \\
\text { s.t. } & \mathbf{q}[1]=\mathbf{q}_{I},\left\|\mathbf{q}[N]-\mathbf{q}_{F}\right\|^{2} \leq\left(\frac{V_{m} T}{N}\right)^{2}, \\
& \|\mathbf{q}[n+1]-\mathbf{q}[n]\|^{2} \leq\left(\frac{V_{m} T}{N}\right)^{2}, n=1, \ldots, N-1, \\
& 0 \leq \varphi_{m}[n] \leq 2 \pi, \forall m, \forall n \\
& \|\mathbf{w}[n]\|^{2} \leq P_{u}, \forall n,
\end{aligned}
$$

where $P_{u}$ is the maximum transmit power of UAV. It is difficult to handle this problem since the optimization variables are coupled in the objective function. To address this issue, the joint problem will be decomposed into three sub-problems and solved iteratively in the next section.

\section{JOINT OPTIMIZATION FOR SECRECY RATE MAXIMIZATION}

In this section, the three variables are optimized in an alternating manner by addressing three sub-problems.

\section{A. Active Beamforming Design for $U A V$}

By fixing $\mathbf{Q}$ and $\boldsymbol{\Psi}$, the transmit beamforming sub-problem can be equivalently reformulated as

$$
\begin{array}{ll}
\max _{\mathbf{w}[n]} & \frac{\left|\widetilde{\mathbf{h}}_{l}[n] \mathbf{w}[n]\right|^{2}+\sigma_{l}^{2}}{\left|\widetilde{\mathbf{h}}_{e}[n] \mathbf{w}[n]\right|^{2}+\sigma_{e}^{2}} \\
\text { s.t. } & \|\mathbf{w}[n]\|^{2} \leq P_{u}, \forall n,
\end{array}
$$

where the concatenated channels are denoted by $\widetilde{\mathbf{h}}_{l}[n]=$ $\mathbf{h}_{u, l}^{H}[n]+\mathbf{h}_{I, l}^{H}[n] \boldsymbol{\Phi}[n] \mathbf{H}_{U I}[n]$ and $\widetilde{\mathbf{h}}_{e}[n]=\mathbf{h}_{u, e}^{H}[n]+$ $\mathbf{h}_{I, e}^{H}[n] \boldsymbol{\Phi}[n] \mathbf{H}_{U I}[n]$. By defining $\mathbf{H}_{l}[n]=\widetilde{\mathbf{h}}_{l}^{H}[n] \widetilde{\mathbf{h}}_{l}[n]$ and $\mathbf{H}_{e}[n]=\widetilde{\mathbf{h}}_{e}^{H}[n] \widetilde{\mathbf{h}}_{e}[n]$, (16) can be rewritten as

$$
\begin{array}{cc}
\max _{\mathbf{w}[n]} & \frac{\mathbf{w}^{H}[n] \mathbf{H}_{l}[n] \mathbf{w}[n]+\sigma_{l}^{2}}{\mathbf{w}^{H}[n] \mathbf{H}_{e}[n] \mathbf{w}[n]+\sigma_{e}^{2}} \\
\text { s.t. } & \mathbf{w}^{H}[n] \mathbf{w}[n] \leq P_{u}, \forall n .
\end{array}
$$

According to [15], the optimal solution to the transmit beamforming is given by $\mathbf{w}_{\text {opt }}[n]=\sqrt{P_{u}} \mathbf{v}_{\max }[n]$, where $\mathbf{v}_{\max }[n]$ is the eigenvector corresponding to the largest eigenvalue of the matrix $\left(\mathbf{H}_{e}[n] P_{u}+\sigma_{e}^{2} \mathbf{I}_{N_{u}}\right)^{-1}\left(\mathbf{H}_{l}[n] P_{u}+\sigma_{l}^{2} \mathbf{I}_{N_{u}}\right)$.

\section{B. Passive Beamforming Design for IRS}

We redefine $\boldsymbol{\Phi}[n]=\operatorname{diag}\left(\theta_{1}[n], \ldots, \theta_{M}[n]\right)$, which has unit modulus constraints. With the optimized active beamforming and given UAV trajectory, the optimal phase shifts of IRS can be obtained by solving

$$
\begin{aligned}
\max _{\boldsymbol{\Phi}[n]} & \frac{\left|\left(\mathbf{h}_{u, l}^{H}[n]+\mathbf{h}_{I, l}^{H}[n] \boldsymbol{\Phi}[n] \mathbf{H}_{U I}[n]\right) \mathbf{w}[n]\right|^{2}+\sigma_{l}^{2}}{\mid\left(\mathbf{h}_{u, e}^{H}[n]+\left.\mathbf{h}_{I, e}^{H}\left[n \boldsymbol{\Phi}[n] \mathbf{H}_{U I}[n]\right) \mathbf{w}[n]\right|^{2}+\sigma_{e}^{2}\right.} \\
\text { s.t. } & \left|\theta_{m}[n]\right|=1, m=1, \ldots, M .
\end{aligned}
$$

Due to the equality $\mathbf{a}^{H} \boldsymbol{\Phi}[n] \mathbf{b}=\mathbf{u}^{H}[n] \operatorname{diag}\left\{\mathbf{a}^{H}\right\} \mathbf{b}$, we have $\mathbf{h}_{I, l}^{H}[n] \boldsymbol{\Phi}[n] \mathbf{H}_{U I}[n] \mathbf{w}[n]=\mathbf{u}^{H}[n] \operatorname{diag}\left\{\mathbf{h}_{I, l}^{H}[n]\right\} \mathbf{H}_{U I}[n] \mathbf{w}[n]$. Introduce an auxiliary vector $\mathbf{u}^{H}[n]=\left[\theta_{1}[n], \ldots, \theta_{M}[n]\right]$, and the objective function can be rewritten as

$$
\max _{\mathbf{u}[n]} \frac{\left|\mathbf{a}_{l}[n]+\mathbf{u}^{H}[n] \mathbf{b}_{l}[n]\right|^{2}+\sigma_{l}^{2}}{\left|\mathbf{a}_{e}[n]+\mathbf{u}^{H}[n] \mathbf{b}_{e}[n]\right|^{2}+\sigma_{e}^{2}},
$$

where $\mathbf{a}_{l}[n]=\mathbf{h}_{u, l}^{H}[n] \mathbf{w}[n], \quad \mathbf{a}_{e}[n]=\mathbf{h}_{u, e}^{H}[n] \mathbf{w}[n]$, $\mathbf{b}_{l}[n]=\operatorname{diag}\left\{\mathbf{h}_{I, l}^{H^{\prime}}[n]\right\} \mathbf{H}_{U I}[n] \mathbf{w}[n], \quad$ and $\mathbf{b}_{e}[n]=$ $\operatorname{diag}\left\{\mathbf{h}_{I, e}^{H}[n]\right\} \mathbf{H}_{U I}[n] \mathbf{w}[n]$. To make it solvable, we further transform (19) into an equivalent form as

$$
\min _{\mathbf{u}[n]} \frac{\left|\mathbf{a}_{e}[n]+\mathbf{u}^{H}[n] \mathbf{b}_{e}[n]\right|^{2}+\sigma_{e}^{2}}{\left|\mathbf{a}_{l}[n]+\mathbf{u}^{H}[n] \mathbf{b}_{l}[n]\right|^{2}+\sigma_{l}^{2}},
$$


which belongs to fractional programming and can be converted into a series of parametric sub-problems as

$$
\begin{array}{ll}
\min _{\mathbf{u}[n]} & \left|\mathbf{a}_{e}[n]+\mathbf{u}^{H}[n] \mathbf{b}_{e}[n]\right|^{2}+\sigma_{e}^{2} \\
& -\eta^{(r-1)}\left(\left|\mathbf{a}_{l}[n]+\mathbf{u}^{H}[n] \mathbf{b}_{l}[n]\right|^{2}+\sigma_{l}^{2}\right) \\
\text { s.t. } & \left|\theta_{m}[n]\right|=1, m=1, \ldots, M,
\end{array}
$$

where $\eta^{(r)}(r \geq 0)$ is a nonnegative parameter with an initial value $\eta^{(0)}=0$, and can be updated by

$$
\eta^{(r)}=\frac{\left|\mathbf{a}_{e}[n]+\mathbf{u}^{(r) H}[n] \mathbf{b}_{e}[n]\right|^{2}+\sigma_{e}^{2}}{\left|\mathbf{a}_{l}[n]+\mathbf{u}^{(r) H}[n] \mathbf{b}_{l}[n]\right|^{2}+\sigma_{l}^{2}} .
$$

$\mathbf{u}^{(r) H}[n]$ is the solution obtained in the $r$ th iteration. However, the objective function in (21a) is still non-convex, and thus, we substitute it with a tractable upper bound as

$$
\begin{aligned}
& \left|\mathbf{a}_{e}[n]+\mathbf{u}^{H}[n] \mathbf{b}_{e}[n]\right|^{2}+\sigma_{e}^{2}-\eta\left(\left|\mathbf{a}_{l}[n]+\mathbf{u}^{H}[n] \mathbf{b}_{l}[n]\right|^{2}+\sigma_{l}^{2}\right) \\
& =\mathbf{u}^{H}[n]\left(\mathbf{b}_{e}[n] \mathbf{b}_{e}^{H}[n]-\mathbf{b}_{l}[n] \mathbf{b}_{l}^{H}[n]\right) \mathbf{w}[n]+\left|\mathbf{a}_{e}[n]\right|^{2}+\sigma_{e}^{2} \\
& -2 \operatorname{Re}\left\{\mathbf{u}^{H}[n]\left(\mathbf{a}_{l}^{*}[n] \mathbf{b}_{l}[n]-\mathbf{a}_{e}^{*}[n] \mathbf{b}_{e}[n]\right)\right\}-\eta\left|\mathbf{a}_{l}[n]\right|^{2}-\eta \sigma_{l}^{2} \\
& \leq \lambda_{\max }(\mathbf{A}[n])\|\mathbf{u}[n]\|^{2}-2 \operatorname{Re}\left\{\mathbf{u}^{H}[n] \mathbf{B}[n]\right\}+\mathbf{C}[n], \quad
\end{aligned}
$$

where $\mathbf{A}[n], \mathbf{B}[n]$ and $\mathbf{C}[n]$ are given by

$$
\begin{aligned}
\mathbf{A}[n]= & \mathbf{b}_{e}[n] \mathbf{b}_{e}^{H}[n]-\eta \mathbf{b}_{l}[n] \mathbf{b}_{l}^{H}[n], \\
\mathbf{B}[n]= & \left(\lambda_{\max }(\mathbf{A}[n]) \mathbf{I}-\mathbf{A}[n]\right) \tilde{\mathbf{u}}[n]+\eta \mathbf{a}_{l}^{*}[n] \mathbf{b}_{l}[n]-\mathbf{a}_{e}^{*}[n] \mathbf{b}_{e}[n], \\
\mathbf{C}[n]= & \tilde{\mathbf{u}}^{H}[n]\left(\lambda_{\max }(\mathbf{A}[n]) \mathbf{I}-\mathbf{A}[n]\right) \tilde{\mathbf{u}}[n] \\
& +\left|\mathbf{a}_{e}[n]\right|^{2}+\sigma_{e}^{2}-\eta\left|\mathbf{a}_{l}[n]\right|^{2}-\eta \sigma_{l}^{2} .
\end{aligned}
$$

Note that $\tilde{\mathbf{u}}[n]$ denotes the optimal solution to $\mathbf{u}[n]$ in the previous iteration. Thus, the objective function in (21a) can be simplified as

$$
\min _{\mathbf{u}[n]} \lambda_{\max }(\mathbf{A}[n])\|\mathbf{u}[n]\|^{2}-2 \operatorname{Re}\left\{\mathbf{u}^{H}[n] \mathbf{B}[n]\right\}
$$

The unit modulus constraint $\left|\theta_{m}[n]\right|=1$ results in $\|\mathbf{u}[n]\|^{2}=M$, which means that $\lambda_{\max }(\mathbf{A}[n])\|\mathbf{u}[n]\|^{2}$ is a constant. It is obvious that the objective function is minimized when $\operatorname{Re}\left\{\mathbf{u}^{H}[n] \mathbf{B}[n]\right\}$ is maximized. Denote the phase of the $m$ th entry of $\mathbf{B}[n]$ as $\mathbf{B}_{m}[n]$, and the optimal phase shift vector can be obtained as

$$
\mathbf{u}_{o p t}^{H}[n]=\left[e^{j \arg \left(\mathbf{B}_{1}[n]\right)}, \ldots, e^{j \arg \left(\mathbf{B}_{M}[n]\right)}\right] .
$$

\section{Optimizing UAV Trajectory with Given Beamforming}

With the transmit beamforming and the phase shift matrix obtained above, the UAV trajectory optimization sub-problem can be separated from (15) as

$$
\begin{aligned}
\max _{\mathbf{Q}} & \frac{1}{N} \sum_{n=1}^{N}\left(R_{L}[n]-R_{E}[n]\right) \\
\text { s.t. } & (15 b) \&(15 c) .
\end{aligned}
$$

Both $R_{L}[n]$ and $R_{E}[n]$ are neither convex nor concave. To deal with $R_{L}[n]$, we make mathematical operations on the received signal power at the legitimate user as

$$
\begin{aligned}
& \left|\left(\mathbf{h}_{u, l}^{H}[n]+\mathbf{h}_{I, l}^{H}[n] \mathbf{\Phi}[n] \mathbf{H}_{U I}[n]\right) \mathbf{w}[n]\right|^{2} \\
& =\frac{\rho}{\sigma^{2}}\left(X_{l}[n] d_{u, l}^{-\alpha}[n]+Y_{l}[n] d_{u, l}^{-\frac{\alpha}{2}}[n] d_{U I}^{-1}[n]+Z_{l}[n] d_{U I}^{-2}[n]\right),
\end{aligned}
$$

where the constant coefficients are derived as

$$
\begin{aligned}
& X_{l}[n]=\widetilde{\mathbf{h}}_{u, l}[n] \mathbf{W}[n] \widetilde{\mathbf{h}}_{u, l}^{H}[n], \\
& Y_{l}[n]=2 \operatorname{Re}\left\{\mathbf{G}_{l}[n] \mathbf{a}_{M}^{T}[n] \mathbf{a}_{N_{U}}[n] \mathbf{W}[n] \widetilde{\mathbf{h}}_{u, l}^{H}[n]\right\}, \\
& Z_{l}[n]=\mathbf{G}_{l}[n] \mathbf{a}_{M}^{T}[n] \mathbf{a}_{N_{U}}[n] \mathbf{W}[n] \mathbf{a}_{N_{U}}^{*}[n] \mathbf{a}_{N_{U}}^{H}[n] \mathbf{G}_{l}^{H}[n] .
\end{aligned}
$$

In (29), $\mathbf{W}[n]=\mathbf{w}[n] \mathbf{w}^{H}[n]$ and $\mathbf{G}_{l}[n]=\mathbf{h}_{I, l}^{H}[n] \mathbf{\Phi}[n]$.

Similarly, the transformation on $R_{E}[n]$ can be given as

$$
\begin{aligned}
& \left|\left(\mathbf{h}_{u, e}^{H}[n]+\mathbf{h}_{I, e}^{H}[n] \mathbf{\Phi}[n] \mathbf{H}_{U I}[n]\right) \mathbf{w}[n]\right|^{2} \\
= & \frac{\rho}{\sigma^{2}}\left(X_{e}[n] d_{u, e}^{-\alpha}[n]+Y_{e}[n] d_{u, e}^{-\frac{\alpha}{2}}[n] d_{U I}^{-1}[n]+Z_{e}[n] d_{U I}^{-2}[n]\right) .
\end{aligned}
$$

where $X_{e}[n], Y_{e}[n]$, and $Z_{e}[n]$ are in the same form to (29).

By introducing auxiliary variables $\mathbf{t}_{1}=\left\{t_{1}[n], \forall n\right\}$ and $\mathbf{t}_{2}=\left\{t_{2}[n], \forall n\right\},(27)$ can be reformulated as

$$
\begin{aligned}
\max _{\substack{\mathbf{Q}, \mathbf{t}_{1}, \mathbf{t}_{\mathbf{2}}, \mathbf{v}_{1}, \mathbf{v}_{2}, \mathbf{v}_{3}}} & \frac{1}{N} \sum_{n=1}^{N}\left(\log _{2}(e) t_{1}[n]-\log _{2}(e) t_{2}[n]\right) \\
\text { s.t. } & 1+\frac{\rho}{\sigma^{2}} D[n] \geq e^{t_{1}[n]}, \\
& 1+\frac{\rho}{\sigma^{2}} E[n] \leq e^{t_{2}[n]}, \\
& (15 b) \&(15 c) .
\end{aligned}
$$

where $D[n]$ and $E[n]$ can be expressed as

$$
\begin{aligned}
& D[n]=X_{l}[n] d_{u, l}^{-\alpha}[n]+Y_{l}[n] d_{u, l}^{-\frac{\alpha}{2}}[n] d_{U I}^{-1}[n]+Z_{l}[n] d_{U I}^{-2}[n], \\
& E[n]=X_{e}[n] d_{u, e}^{-\alpha}[n]+Y_{e}[n] d_{u, e}^{-\frac{\alpha}{2}}[n] d_{U I}^{-1}[n]+Z_{e}[n] d_{U I}^{-2}[n] .
\end{aligned}
$$

Particularly, $D[n]$ and $E[n]$ should be converted into a concave and a convex ones, respectively.

For convenience, we define several functions as

$$
\begin{aligned}
& F_{x}[n]=X_{l}[n] d_{u, l}^{-\alpha}[n], \quad F_{z}[n]=Z_{l}[n] d_{U I}^{-2}[n], \\
& F_{y}[n]=Y_{l}[n] d_{u, l}^{-\frac{\alpha}{2}}[n] d_{U I}^{-1}[n] .
\end{aligned}
$$

First, we introduce slack variables $\mathbf{v}_{1}=\left\{v_{1}[n], \forall n\right\}$ and $\mathbf{v}_{3}=\left\{v_{3}[n], \forall n\right\}$, which satisfy

$$
d_{u, l}[n] \leq v_{1}[n], \quad d_{U I}[n] \leq v_{3}[n], \forall n .
$$

Since $v_{1}^{2}[n]$ is convex, with the given local point $\bar{v}_{1}[n]$, its first-order Taylor expansion can be expressed as

$$
\begin{aligned}
v_{1}^{2}[n] & \geq \bar{v}_{1}^{2}[n]+2 \bar{v}_{1}[n]\left(v_{1}[n]-\bar{v}_{1}[n]\right) \\
& =-\bar{v}_{1}^{2}[n]+2 \bar{v}_{1}[n] v_{1}[n] .
\end{aligned}
$$

According to (35), we have $d_{u, l}[n]^{2}-v_{1}[n]^{2} \leq 0$. Therefore, based on (36) we have

$$
d_{u, l}^{2}[n]+\bar{v}_{1}^{2}[n]-2 \bar{v}_{1}[n] v_{1}[n] \leq 0, \forall n .
$$

Due to the fact that $\mathbf{W}[n]=\mathbf{w}[n] \mathbf{w}^{H}[n]$ is generally a 
positive semi-definite matrix which holds $\mathbf{W}[n] \succeq 0$, we have $X_{l}[n] \geq 0$ and $Z_{l}[n] \geq 0$. Replace $d_{u, l}[n]$ and $d_{U I}[n]$ with $v_{1}[n]$ and $v_{3}[n]$, respectively, we have

$$
F_{x}[n] \geq X_{l}[n] v_{1}^{-\alpha}[n], \quad F_{z}[n] \geq Z_{l}[n] v_{3}^{-2}[n],
$$

which are convex with $v_{1}[n]$ and $v_{3}[n]$. Hence, $F_{x}[n]$ and $F_{z}[n]$ are lower bounded by the corresponding first-order Taylor series of the right-side terms as

$$
\begin{aligned}
F_{x}[n] & \geq(1+\alpha) X_{l}[n] \bar{v}_{1}^{-\alpha}[n]-\alpha X_{l}[n] \bar{v}_{1}^{-\alpha-1}[n] v_{1}[n] \\
& \triangleq \mathcal{L}_{x}\left(\bar{v}_{1}[n], v_{1}[n]\right) \\
F_{z}[n] & \geq 3 Z_{l}[n] \bar{v}_{3}^{-2}[n]-2 Z_{l}[n] \bar{v}_{3}^{-3}[n] v_{3}[n] \\
& \triangleq \mathcal{L}_{z}\left(\bar{v}_{3}[n], v_{3}[n]\right) .
\end{aligned}
$$

Nevertheless, $Y_{l}[n]$ is not necessarily positive or negative. When $Y_{l}[n] \geq 0$, through utilizing slack variables $v_{1}[n]$ and $v_{3}[n], F_{y}[n]$ can be approximated by

$$
\begin{aligned}
& F_{y}[n] \geq\left(2+\frac{\alpha}{2}\right) Y_{l}[n] \bar{v}_{1}^{-\frac{\alpha}{2}}[n] \bar{v}_{3}^{-1}[n] \\
& -\frac{\alpha}{2} Y_{l}[n] \bar{v}_{3}^{-1}[n] \bar{v}_{1}^{-\frac{\alpha}{2}-1}[n] v_{1}[n]-Y_{l}[n] \bar{v}_{1}^{-\frac{\alpha}{2}}[n] \bar{v}_{3}^{-2}[n] v_{3}[n] \\
& \triangleq \mathcal{L}_{y}\left(\bar{v}_{1}[n], \bar{v}_{3}[n], v_{1}[n], v_{3}[n]\right) .
\end{aligned}
$$

In contrast, if $Y_{l}[n]<0$, the above operations do not make sense anymore. We introduce another two relaxation variables $\mathbf{V}_{1}=\left\{V_{1}[n], \forall n\right\}$ and $\mathbf{V}_{3}=\left\{V_{3}[n], \forall n\right\}$ as

$$
V_{1}[n] \leq d_{u, l}[n], \quad V_{3}[n] \leq d_{U I}[n], \forall n .
$$

In this case, $Y_{l}[n] V_{1}^{-\frac{\alpha}{2}}[n] V_{3}^{-1}[n]$ serves as a lower bound for $F_{y}[n]$. Then, with the given local points $\bar{V}_{1}[n]$ and $\bar{V}_{3}[n]$, the alteration of $F_{y}[n]$ can be given as

$$
\begin{aligned}
F_{y}[n] & \geq Y_{l}[n] V_{1}^{-\frac{\alpha}{2}}[n] V_{3}^{-1}[n] \\
& =\frac{Y_{l}[n]}{2}\left[\left(V_{1}^{-\frac{\alpha}{2}}[n]+V_{3}^{-1}[n]\right)^{2}-V_{1}^{-\alpha}-V_{3}^{-2}[n]\right] \\
& \geq \frac{Y_{l}[n]}{2}\left[\left(V_{1}^{-\frac{\alpha}{2}}[n]+V_{3}^{-1}[n]\right)^{2}-(1+\alpha) \bar{V}_{1}^{-\alpha}[n]\right. \\
& \left.+\alpha \bar{V}_{1}^{-\alpha-1}[n] V_{1}[n]-3 \bar{V}_{3}^{-2}[n]+2 \bar{V}_{3}^{-3}[n] V_{3}[n]\right] \\
& \triangleq \mathcal{T}_{y}\left(\bar{V}_{1}[n], \bar{V}_{3}[n], V_{1}[n], V_{3}[n]\right),
\end{aligned}
$$

$\mathcal{T}_{y}$ can be easily proved to be concave under the condition $F_{y}[n]<0$ by using its Hessian matrix.

According to (42), we have $V_{1}^{2}[n]-d_{u, l}^{2}[n] \leq 0$, where $-d_{u, l}^{2}[n]=-\left\|\mathbf{q}[n]-\mathbf{C}_{l}\right\|^{2}-H_{u}^{2}$. Since the negative normsquared function $-\left\|\mathbf{q}[n]-\mathbf{C}_{l}\right\|^{2}$ is concave with respect to the variable vector $\mathbf{q}[n]$, it has a convex upper bound as

$$
\begin{aligned}
& -\left\|\mathbf{q}[n]-\mathbf{C}_{l}\right\|^{2} \stackrel{(b)}{\leq}-\left\|\mathbf{q}^{r}[n]-\mathbf{C}_{l}\right\|^{2}-2\left(\mathbf{q}^{r}[n]-\mathbf{C}_{l}\right)^{T}\left(\mathbf{q}[n]-\mathbf{q}^{r}[n]\right) \\
& =\left\|\mathbf{q}^{r}[n]\right\|^{2}-2\left(\mathbf{q}^{r}[n]-\mathbf{C}_{l}\right)^{T} \mathbf{q}[n]-\left\|\mathbf{C}_{l}\right\|^{2},
\end{aligned}
$$

where $\mathbf{q}^{r}[n]$ denotes the given point and $\mathbf{Q}^{r} \triangleq\left\{\mathbf{q}^{r}[n], \forall n\right\}$ denotes the trajectory of UAV in the $r$ th iteration. Thus, substituting $-d_{u, l}^{2}[n]$ with its upper bound, we have

$$
V_{1}^{2}[n]+\left\|\mathbf{q}^{r}[n]\right\|^{2}-2\left(\mathbf{q}^{r}[n]-\mathbf{C}_{l}\right)^{T} \mathbf{q}[n]-\left\|\mathbf{C}_{l}\right\|^{2}+H_{u}^{2} \leq 0 .
$$

Define a binary variable $I_{y}[n]$, which indicates $Y_{l}[n] \geq 0$ in the $n$th time slot if $I_{y}[n]=1$; otherwise, $I_{y}[n]=0$. The concave lower bound for $D[n]$ can be obtained as

$$
\begin{aligned}
& D[n]=F_{x}[n]+F_{y}[n]+F_{z}[n] \\
& \quad \geq \mathcal{L}_{x}[n]+\mathcal{L}_{z}[n]+I_{y}[n] \mathcal{L}_{y}[n]+\left(1-I_{y}[n]\right) \mathcal{T}_{y}[n] \\
& \quad \triangleq \widetilde{D}[n] .
\end{aligned}
$$

In (31c), $e^{t_{2}[n]}$ is convex and hence is approximated by its first-order Taylor expansion at a given feasible point $\bar{t}_{2}[n]$.

$$
e^{t_{2}[n]} \geq e^{\bar{t}_{2}[n]}\left(t_{2}[n]-\bar{t}_{2}[n]+1\right) .
$$

Similar to the operations on $D[n]$, new functions related to $E[n]$ are defined as

$$
\begin{array}{ll}
\mathcal{F}_{x}[n]=X_{e}[n] d_{u, e}^{-\alpha}[n], & \mathcal{F}_{z}[n]=Z_{e}[n] d_{U I}^{-2}[n], \\
\mathcal{F}_{y}[n] & =Y_{e}[n] d_{u, e}^{-\frac{\alpha}{2}}[n] d_{U I}^{-1}[n] .
\end{array}
$$

Contrary to $D[n]$ that needs a lower bound, $E[n]$ ought to be approximated by a convex upper bound. Introduce auxiliary variables $\mathbf{v}_{2}=\left\{v_{2}[n], \forall n\right\}$ and $\mathbf{V}_{2}=\left\{V_{2}[n], \forall n\right\}$ as

$$
d_{u, e}[n] \leq v_{2}[n], \quad V_{2}[n] \leq d_{u, e}[n], \forall n .
$$

To cope with $\mathcal{F}_{x}[n]$ and $\mathcal{F}_{z}[n]$, we utilize $V_{2}[n]$ and $V_{3}[n]$ to replace $d_{u, e}[n]$ and $d_{U I}[n]$, respectively, to obtain their upper approximations as

$$
\begin{aligned}
& \mathcal{F}_{x}[n] \leq X_{e}[n] V_{2}^{-\alpha}[n] \triangleq \mathcal{S}_{x}[n], \\
& \mathcal{F}_{z}[n] \leq Z_{e}[n] V_{3}^{-2}[n] \triangleq \mathcal{S}_{z}[n],
\end{aligned}
$$

which are convex. Moreover, when $Y_{e}[n] \geq 0, \mathcal{F}_{y}[n]$ is converted into a convex counterpart as

$$
\begin{aligned}
& \mathcal{F}_{y}[n] \leq \frac{Y_{e}[n]}{2}\left[\left(V_{2}^{-\frac{\alpha}{2}}[n]+V_{3}^{-1}[n]\right)^{2}-(1+\alpha) \bar{V}_{2}^{-\alpha}[n]\right. \\
& \left.+\alpha \bar{V}_{2}^{-\alpha-1}[n] V_{2}[n]-3 \bar{V}_{3}^{-2}[n]+2 \bar{V}_{3}^{-3}[n] V_{3}[n]\right] \\
& \triangleq \mathcal{S}_{y}\left(\bar{V}_{2}[n], \bar{V}_{3}[n], V_{2}[n], V_{3}[n]\right),
\end{aligned}
$$

where $\bar{V}_{2}[n]$ and $\bar{V}_{3}[n]$ are the given local points.

In the other case when $Y_{e}[n]<0, \mathcal{F}_{y}[n]$ can be approximated by its upper-bound function concerning $v_{2}[n]$ and $v_{3}[n]$. Specifically, it is transformed into a convex one as

$$
\begin{aligned}
& \mathcal{F}_{y}[n] \leq\left(2+\frac{\alpha}{2}\right) Y_{e}[n] \bar{v}_{2}^{-\frac{\alpha}{2}}[n] \bar{v}_{3}^{-1}[n] \\
& -\frac{\alpha}{2} Y_{e}[n] \bar{v}_{3}^{-1}[n] \bar{v}_{2}^{-\frac{\alpha}{2}-1}[n] v_{2}[n]-Y_{e}[n] \bar{v}_{2}^{-\frac{\alpha}{2}}[n] \bar{v}_{3}^{-2}[n] v_{3}[n] \\
& \triangleq \mathcal{Q}_{y}\left(\bar{v}_{2}[n], \bar{v}_{3}[n], v_{2}[n], v_{3}[n]\right),
\end{aligned}
$$

where $\bar{v}_{2}[n]$ and $\bar{v}_{3}[n]$ denote the given feasible points.

Using $\mathcal{I}_{y}[n]=1$ to imply $Y_{e}[n] \geq 0, E[n]$ can be approximated as

$$
\begin{aligned}
E[n] & =\mathcal{F}_{x}[n]+\mathcal{F}_{y}[n]+\mathcal{F}_{z}[n] \\
& \leq \mathcal{S}_{x}[n]+\mathcal{S}_{z}[n]+\mathcal{I}_{y}[n] \mathcal{S}_{y}[n]+\left(1-\mathcal{I}_{y}[n]\right) \mathcal{Q}_{y}[n] \\
& \triangleq \widetilde{E}[n] .
\end{aligned}
$$

After the aforementioned transformations, the original con- 
straint (31c) can be rewritten as

$$
1+\frac{\rho}{\sigma^{2}} \widetilde{E}[n] \leq e^{\bar{t}_{2}[n]}\left(t_{2}[n]-\bar{t}_{2}[n]+1\right),
$$

which is convex now.

As a result, the trajectory optimization problem can be recasted into a convex problem as

$$
\begin{aligned}
\underset{\substack{\mathbf{a}_{\mathbf{Q}}, \mathbf{t}_{1}, \mathbf{t}_{2}, \mathbf{v}_{1}, \mathbf{v}_{2}, \mathbf{v}_{3} \\
\mathbf{V}_{1}, \mathbf{V}_{2}, \mathbf{V}_{3}}}{ } & \frac{1}{N} \sum_{n=1}^{N} t_{1}[n]-t_{2}[n] \\
\text { s.t. } & 1+\frac{\rho}{\sigma^{2}} \widetilde{D}[n] \geq e^{t_{1}[n]}, \\
& 1+\frac{\rho}{\sigma^{2}} \widetilde{E}[n] \leq e^{\bar{t}_{2}[n]}\left(t_{2}[n]-\bar{t}_{2}[n]+1\right), \\
& d_{u, l}^{2}[n]+\bar{v}_{1}^{2}[n]-2 \bar{v}_{1}[n] v_{1}[n] \leq 0, \forall n, \\
& d_{u, e}^{2}[n]+\bar{v}_{2}^{2}[n]-2 \bar{v}_{2}[n] v_{2}[n] \leq 0, \forall n, \\
& d_{U I}^{2}[n]+\bar{v}_{3}^{2}[n]-2 \bar{v}_{3}[n] v_{3}[n] \leq 0, \forall n . \\
& V_{1}^{2}[n]+\left\|\mathbf{q}^{r}[n]\right\|^{2}-2\left(\mathbf{q}^{r}[n]-\mathbf{C}_{l}\right)^{T} \mathbf{q}[n] \\
& -\left\|\mathbf{C}_{l}\right\|^{2}+H_{u}^{2} \leq 0, \\
& V_{2}^{2}[n]+\left\|\mathbf{q}^{r}[n]\right\|^{2}-2\left(\mathbf{q}^{r}[n]-\mathbf{C}_{e}\right)^{T} \mathbf{q}[n] \\
& -\left\|\mathbf{C}_{e}\right\|^{2}+H_{u}^{2} \leq 0, \\
& V_{3}^{2}[n]+\left\|\mathbf{q}^{r}[n]\right\|^{2}-2\left(\mathbf{q}^{r}[n]-\mathbf{C}_{I}\right)^{T} \mathbf{q}[n] \\
& -\left\|\mathbf{C}_{I}\right\|^{2}+H_{u I}^{2} \leq 0, \\
& (15 a) \&(15 b) .
\end{aligned}
$$

which can be solved efficiently by existing optimization tools.

\section{Overall Algorithm}

An effective algorithm is proposed to further enhance the network security by solving the three sub-problems alternately until convergence. With fixed UAV trajectory and IRS phase shifts, the optimal transmit beamforming can be obtained directly, while the solutions to the passive beamforming and UAV trajectory are updated by solving the approximate problems converted from the non-convex ones in each iteration. The detailed procedure of the iterative algorithm is summarized in Algorithm 1.

\section{Simulation Results}

In this section, simulations are conducted to evaluate the performance of the proposed design for secure IRSassisted UAV networks. Unless stated otherwise, the default simulation parameters are set as: $H_{u}=100 \mathrm{~m}, V_{m}=20 \mathrm{~m} / \mathrm{s}$, $H_{I}=30 \mathrm{~m}, N_{u}=16, M=64, P_{u}=20 \mathrm{dBm}, \rho_{0}=-30$ $\mathrm{dB}$, and $\sigma_{l}^{2}=\sigma_{e}^{2}=-80 \mathrm{dBm}$. The path-loss exponents are $\alpha=3.5$ and $\beta=2.4$. Assume the IRS element spacing and antenna separation to be $\widetilde{d}_{x}=\widetilde{d}_{z}=\frac{\lambda}{4}$ and $\widetilde{d}=\frac{\lambda}{2}$. The coordinate system is set with the origin at the location of IRS. The legitimate user is assumed to be located at $\mathbf{C}_{l}=[20,30]^{T}$ $\mathrm{m}$, and the initial and final horizontal coordinates of UAV are set as $\mathbf{q}_{I}=[-300,80]^{T} \mathrm{~m}$ and $\mathbf{q}_{F}=[300,80]^{T} \mathrm{~m}$, respectively. The eavesdropper is randomly generated and located close to the legitimate user.
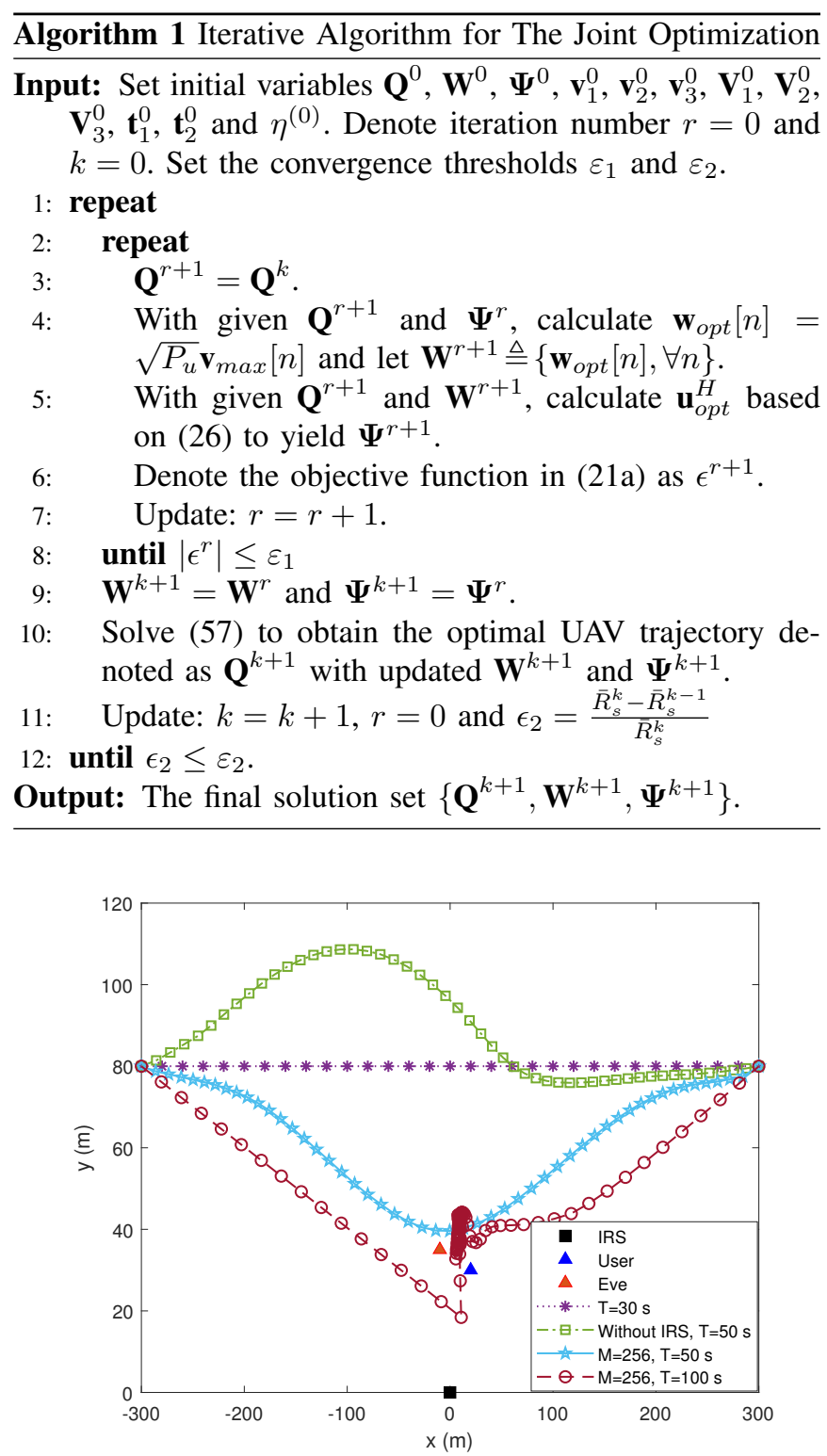

Fig. 2. The trajectories of UAV in different cases of the proposed scheme and the benchmark without IRS.

First, Fig. 2 plots the optimal trajectories of UAV under different cases. With the given initial and final locations, one can see that $T=30 \mathrm{~s}$ is the minimum time required for the UAV to fly straight from the start to the destination. For the case without IRS, it is shown that the UAV tends to be away from the eavesdropper to avoid being wiretapped. In contrast, the UAV flies close to the users when $T=50 \mathrm{~s}$. When $T=100 \mathrm{~s}$, the UAV flies directly towards a location between the user and the IRS, spends a large amount of time near the user and finally flies to the destination. This is because the received power of reflecting signal at the user can be enhanced by passive beamforming when the UAV is close to the IRS and the strength of direct link can also be improved by approaching the user.

In Fig. 3, we compare the proposed scheme with the other 


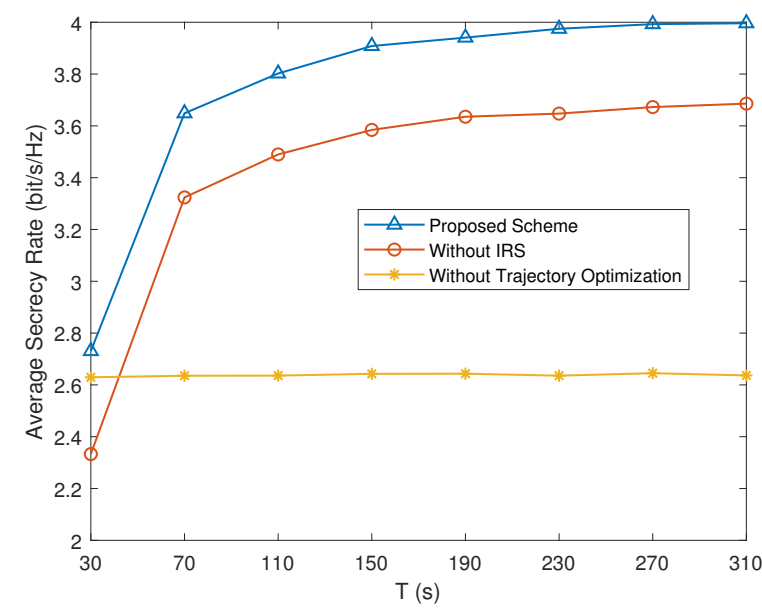

Fig. 3. Average secrecy rate versus the flying period $T$.

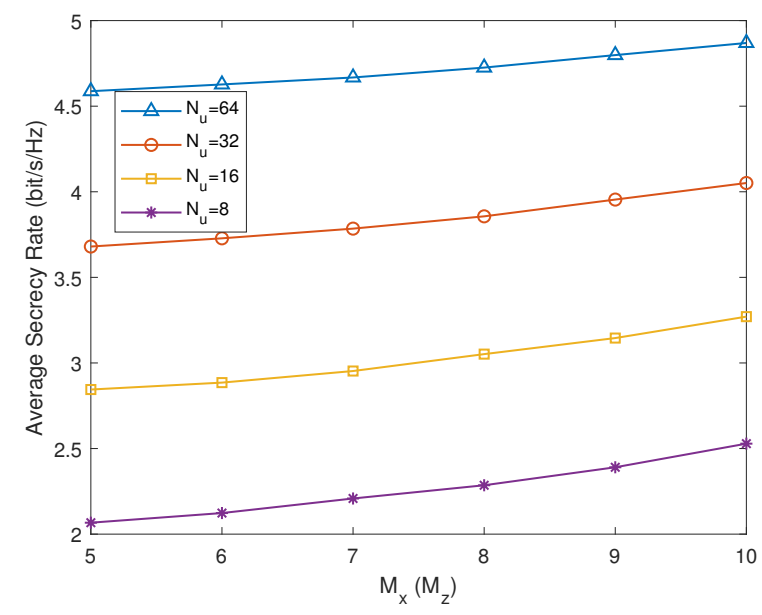

Fig. 4. Average secrecy rate versus different number of reflecting elements.

two benchmarks regarding the average secrecy rate when considering different $T$. One can see that the secrecy rate of both the proposed scheme and the optimization scheme without IRS (passive beamforming) increases with $T$ but gradually becomes saturated. The secrecy rate is stable with different values of $T$ if no trajectory optimization is applied, due to the fact that the UAV moves uniformly between the start and the end in a straight line. More importantly, the superiority of the proposed scheme is demonstrated by the performance gap with the other two benchmarks.

Furthermore, we plot the average secrecy rate versus the number of reflecting elements in Fig. 4, with $M_{x}=M_{z}$. As expected, the results show that the average secrecy rate increases with $M_{x}\left(M_{z}\right)$. This is because with a larger size of IRS, the passive beamforming gain of the legitimate user is able to be more benefited without resulting in the improvement of the eavesdropping power. In addition, we can observe that equipping more antennas at the UAV also contributes to the security performance. Thus, jointly deploying large-scale antennas and reflecting elements can cooperatively achieve a higher secrecy rate.

\section{CONCLUSions}

In this paper, the secrecy rate maximization problem has been studied for IRS-assisted UAV wireless networks, where the transmit and passive beamforming and the UAV trajectory are jointly optimized. Due to the fact that the joint optimization problem is non-convex and intractable, we decompose it into three sub-problems and propose an iterative algorithm to solve them alternately. The effectiveness of the proposed algorithm is demonstrated by the numerical results.

\section{REFERENCES}

[1] Y. Zeng, R. Zhang, and T. J. Lim, "Wireless communications with unmanned aerial vehicles: Opportunities and challenges," IEEE Commun. Mag., vol. 54, no. 5, pp. 36-42, May 2016.

[2] Y. Zeng and R. Zhang, "Energy-efficient UAV communication with trajectory optimization," IEEE Trans. Wireless Commun., vol. 16, no. 6, pp. 3747-3760, Jun. 2017.

[3] X. Jiang, M. Sheng, N. Zhao, C. Xing, W. Lu, and X. Wang, "Green UAV communications for 6G: A survey," Chinese J. Aeronaut., to appear.

[4] X. Pang, J. Tang, N. Zhao, X. Zhang, and Y. Qian, "Energy-efficient design for mmwave-enabled NOMA-UAV networks," Sci. China Inf. Sci., vol. 64, no. 4, p. 140303, Mar. 2021.

[5] Y. Zeng, J. Lyu, and R. Zhang, "Cellular-connected UAV: Potential, challenges, and promising technologies," IEEE Wireless Commun., vol. 26, no. 1, pp. 120-127, Feb. 2019.

[6] A. Al-Hourani, S. Kandeepan, and S. Lardner, "Optimal LAP altitude for maximum coverage," IEEE Wireless Commun. Lett., vol. 3, no. 6, pp. 569-572, Dec. 2014

[7] M. Mozaffari, W. Saad, M. Bennis, Y. H. Nam, and M. Debbah, "A tutorial on UAVs for wireless networks: Applications, challenges, and open problems," IEEE Comm. Surveys Tuts., vol. 21, no. 3, pp. 2334 2360, 3rd Quart. 2019.

[8] Q. Wu and R. Zhang, "Towards smart and reconfigurable environment: Intelligent reflecting surface aided wireless network," IEEE Commun. Mag., vol. 58, no. 1, pp. 106-112, Jan. 2020.

[9] Q. Wu, S. Zhang, B. Zheng, C. You, and R. Zhang, "Intelligent reflecting surface aided wireless communications: A tutorial," IEEE Trans. Commun., vol. 69, no. 5, pp. 3313-3351, May 2021.

[10] S. Alfattani, W. Jaafar, Y. Hmamouche, H. Yanikomeroglu, A. Yonga çoglu, N. D. Dào, and P. Zhu, "Aerial platforms with reconfigurable smart surfaces for 5G and beyond," IEEE Commun. Mag., vol. 59, no. 1, pp. 96-102, Jan. 2021.

[11] S. Li, B. Duo, X. Yuan, Y. Liang, and M. Di Renzo, "Reconfigurable intelligent surface assisted UAV communication: Joint trajectory design and passive beamforming," IEEE Wireless Commun. Lett., vol. 9, no. 5, pp. 716-720, May 2020.

[12] L. Ge, P. Dong, H. Zhang, J. Wang, and X. You, "Joint beamforming and trajectory optimization for intelligent reflecting surfaces-assisted UAV communications," IEEE Access, vol. 8, pp. 78702-78712, 2020.

[13] Z. Wei, Y. Cai, Z. Sun, D. W. K. Ng, J. Yuan, M. Zhou, and L. Sun, "Sum-rate maximization for IRS-assisted UAV OFDMA communication systems," IEEE Trans.. Wireless Commun., vol. 20, no. 4, pp. 2530-2550, Apr. 2021

[14] S. Fang, G. Chen, and Y. Li, "Joint optimization for secure intelligent reflecting surface assisted UAV networks," IEEE Wireless Commun. Lett., vol. 10, no. 2, pp. 276-280, Feb. 2021.

[15] Q. Li and W. Ma, "Optimal and robust transmit designs for MISO channel secrecy by semidefinite programming," IEEE Trans. Signal Process., vol. 59, no. 8, pp. 3799-3812, Aug. 2011. 